\title{
How important is it to control nocturnal hypertension with angiotensin II type 1 receptor blockers?
}

\author{
Shin-ichiro Miura and Keijiro Saku \\ Hypertension Research (2013) 36, 194-195; doi:10.1038/hr.2012.188; published online 22 November 2012
}

$\mathrm{B}$ etter blood pressure (BP) control is associated with remarkable clinical benefits with regard to cardiovascular $(\mathrm{CV})$ and renal protection. Patients with chronic kidney disease $(\mathrm{CKD})$ are at significantly higher risk of $\mathrm{CV}$ disease (CVD), ${ }^{1}$ and patients with overt proteinuria as well as albuminuria without a reduction in the estimated glomerular filtration rate (eGFR) are also at significantly higher risk. ${ }^{1}$ In addition, proteinuria or albuminuria itself should be a target for reducing hard end points. Angiotensin II (Ang II) type $1\left(\mathrm{AT}_{1}\right)$ receptor blockers (ARBs) are highly selective for the $\mathrm{AT}_{1}$ receptor and block the deleterious effects of Ang II. ${ }^{2}$ ARBs clearly decrease proteinuria and protect the kidneys. ${ }^{3}$ A nocturnal increase in $\mathrm{BP}$ on ambulatory monitoring is superior to office BP for predicting a worsening of albuminuria in elderly individuals with type 2 diabetes. ${ }^{4}$ Non-dippers have been shown to have a greater risk of $\mathrm{CV}$ than dippers. With regard to morning $\mathrm{BP}$, morning hypertension (HT) can lead to progressive target organ damage and trigger CV events. ${ }^{5}$ It is still controversial whether treatment with ARB is more effective for all types of HT, such as nocturnal and morning HT, or in patients with albuminuria compared with conventional therapy without $\mathrm{ARBs}$ in patients with CKD.

In this issue of Hypertension Research, Yanagi et al. ${ }^{6}$ reported that add-on therapy

Shin-ichiro Miura and K Saku are at Department of Cardiology, Fukuoka University School of Medicine, Fukuoka, Japan; Shin-ichiro Miura and K Saku are at Department of Molecular Cardiovascular Therapeutics, Fukuoka University School of Medicine, Fukuoka, Japan and Shin-ichiro Miura is at Department of Molecular Cardiology, Cleveland Clinic Foundation, Cleveland, $\mathrm{OH}$, USA

E-mail: miuras@cis.fukuoka-u.ac.jp with the ARB olmesartan improves the ambulatory BP profile by preferential reduction of the nighttime $\mathrm{BP}$, with the concomitant inhibition of renal injury in hypertensive patients with $\mathrm{CKD}$, although the mean levels of systolic $\mathrm{BP} /$ diastolic $\mathrm{BP}$ (SBP/DBP) at night after treatment in the olmesartan group $(121 / 70 \mathrm{~mm} \mathrm{Hg})$ were comparable to the values in the non- $A R B$ group $(124 / 70 \mathrm{~mm} \mathrm{Hg})$ according to the ambulatory BP profile. In addition, there were significant positive associations between the after-treatment/baseline (A/B) ratio of nighttime SBP and that of the urinary protein excretion rate (UPCR), and between the A/B ratio of nighttime SBP and that of the urinary albumin excretion rate (UACR). The decreases in UPCR and UACR were at least in part dependent on the reduction of nighttime SBP, as ARBs dilate efferent arterioles directly, in addition to their BP-lowering effects, and reduce intraglomerular pressure. Consequently, ARBs decrease UPCR and prevent the progression of renal dysfunction.

Recent clinical and basic studies have demonstrated that not all ARBs have the same effects and some benefits conferred by ARBs may be molecule-specific effects, rather than class effects. ${ }^{7}$ Olmesartan significantly decreased $\mathrm{BP}$, proteinuria and the rate of change of reciprocal serum creatinine in a randomized, placebo-controlled multicenter clinical trial, ORIENT (Olmesartan Reducing Incidence of End stage Renal Disease in Diabetic Nephropathy Trial). ${ }^{8}$ Although the renoprotective effects of ARBs may be class effects, the present study clearly showed that the reduction of nighttime SBP by olmesartan is critical for inducing a renoprotective effect. Olmesartan has been reported to exert a longer-lasting BP- lowering effect due to the fact that its structure contains a characteristic 'doublechain domain.' Although olmesartan was added in the morning in this study, some ingenuity may also be required to achieve the target nighttime BP, such as the administration of an antihypertensive agent at bedtime. Olmesartan has been shown to improve the altered ambulatory BP profile in $\mathrm{CKD}$ even when it is administered only once in the morning. ${ }^{10}$ Thus, olmesartan add-on treatment might also be useful in this respect. Moreover, there was a trend toward a decrease in the $\mathrm{A} / \mathrm{B}$ ratios of morning SBP and DBP in the olmesartan add-on group compared with the non-ARB group, although the differences were not significant. Antihypertensive treatment with a target morning BP of $<135 / 85 \mathrm{~mm} \mathrm{Hg}$ leads to more effective protection than conventional antihypertensive treatment based on the office BP. ${ }^{10}$

Yanagi et al. ${ }^{6}$ also analyzed the effects of olmesartan add-on therapy on markers of inflammation, the renal renin-angiotensin system, oxidative stress and fibrosis. Interestingly, the $\mathrm{A} / \mathrm{B}$ ratio of urinary type IV collagen, which is an important marker for renal injury, after treatment in the olmesartan add-on group was significantly suppressed compared with that in the nonARB group. Although the ratio of urinary type IV collagen to creatinine was not identified as a factor that was associated with a low eGFR in a cross-sectional community-based study, ${ }^{11}$ the excretion of urinary type IV collagen has been shown to be increased in diabetic patients with albuminuria. $^{12}$ As treatment with other ARBs, such as telmisartan, has also been shown to significantly reduce urinary protein and type IV collagen, ${ }^{13}$ and as 
valsartan lowered UAER more effectively than amlodipine in patients with type 2 diabetes, ${ }^{14}$ Yanagi et $a l .{ }^{6}$ confirmed this ability of ARBs. They found a significant positive relationship between the $\mathrm{A} / \mathrm{B}$ ratio of nighttime SBP and that of urinary type IV collagen. ${ }^{6}$ This finding is very important for explaining why the reduction of nighttime SBP was associated with a decrease in proteinuria or albuminuria and subsequently induced renal protection.

Another important issue raised by Yanagi et al. ${ }^{6}$ was that the percentage of angiotensin converting enzyme inhibitor (ACEI) prescribed in the non-ARB group (57\%) was about fourfold greater than that in the olmesartan add-on group (14\%), ${ }^{6}$ as ACEI also hass a role in protecting against renal dysfunction. ${ }^{15}$ Although the authors explain that UPCR and UACR after treatment in ACEI ( -$)$ patients was greater than that in ACEI $(+)$ patients in the non-ARB group, which also supports the notion that ACEI has renoprotective effects in hypertensive CKD patients, further investigation will be needed to resolve this issue.

In conclusion, clinical trials, including that of Yanagi et al., ${ }^{6}$ have shown that ARBs decrease UPCR and UACR with a reduction of urinary type IV collagen. Importantly, olmesartan induced a reduction of nighttime SBP. The benefits conferred by ARBs may be class effects, and we must be careful when comparing their results and interpreting their clinical impact.

\section{CONFLICT OF INTEREST}

The authors declare no conflict of interest.

1 Chronic Kidney Disease Prognosis Consortium, Matsushita K, van der Velde M, Astor BC, Woodward M, Levey AS, de Jong PE, Coresh J, Gansevoort RT. Association of estimated glomerular filtration rate and albuminuria with all-cause and cardiovascular mortality in general population cohorts: a collaborative metaanalysis. Lancet 2010; 375: 2073-2081.

2 Gasparo M, Catt KJ, Inagami T, Wright JW, Unger T. International Union of Pharmacology. XXIII. The angiotensin II receptors. Pharmacol Rev 2000; 52: 415-472.

3 Parving $\mathrm{HH}$, Lehnert $\mathrm{H}$, Bröchner-Mortensen J, Gomis $\mathrm{R}$, Andersen S, Arner P. Irbesartan in Patients with Type 2 Diabetes and Microalbuminuria Study Group. The effect of irbesartan on the development of diabetic nephropathy in patients with type 2 diabetes. $N$ Eng/ J Med 2001; 345: 870-878.

4 Palmas W, Pickering T, Teresi J, Schwartz JE, Eguchi K, Field L, Weinstock RS, Shea S. Nocturnal blood pressure elevation predicts progression of albuminuria in elderly people with type 2 diabetes. J Clin Hypertens (Greenwich) 2008; 10: 12-20.

5 Kario K, White WB. Early morning hypertension: what does it contribute to overall cardiovascular risk assessment? J Am Soc Hypertens 2008; 2: 397-402.

6 Yanagi M, Tamura K, Fujikawa T, Wakui H, Kanaoka T, Ohsawa M, Azushima K, Maeda A, Kobori H, Umemura $\mathrm{S}$. The angiotensin II type 1 receptor blocker olmesartan preferentially improves nocturnal hypertension and proteinuria in chronic kidney disease. Hypertens Res 2013; 36: 262-269.
7 Miura S, Karnik SS, Saku K. Angiotensin II type 1 receptor blockers: Class effects vs. Molecular effects. J Renin Angiotensin Aldosterone Syst 2011; 12: 1-7.

8 Imai E, Chan JC, Ito S, Yamasaki T, Kobayashi F, Haneda M, Makino H. ORIENT study investigators. Effects of olmesartan on renal and cardiovascular outcomes in type 2 diabetes with overt nephropathy: a multicentre, randomised, placebo-controlled study. Diabetologia 2011; 54: 2978-2986.

9 Kiya Y, Miura S, Fujino M, Imaizumi S, Karnik SS, Saku K. Clinical and pharmacotherapeutic relevance of the double-chain domain of the angiotensin II type 1 receptor blocker olmesartan. Clin Exp Hypertens 2010; 32: 129-136.

10 Smolensky MH, Hermida RC, Portaluppi F. Comparison of the efficacy of morning versus evening administration of olmesartan in uncomplicated essential hypertension. Chronobiol Int 2007; 24: 171-181.

11 Takamatsu N, Abe $\mathrm{H}$, Tominaga T, Nakahara K, Ito $\mathrm{Y}$, Okumoto Y, Kim J, Kitakaze M, Doi T. Risk factors for chronic kidney disease in Japan: a community-based study. BMC Nephrol 2009; 10: 34.

12 Cohen MP, Lautenslager GT, Shearman CW. Increased Collagen IV Excretion in Diabetes. A marker of compromised filtration function. Diabetes Care 2001; 24: 914-918.

13 Yamamoto T, Nakagawa T, Suzuki H, Ohashi N, Fukasawa H, Fujigaki Y, Kato A, Nakamura Y, Suzuki $F$, Hishida $A$. Urinary angiotensinogen as a marker of intrarenal angiotensin II activity associated with deterioration of renal function in patients with chronic kidney disease. J Am Soc Nephrol 2007; 18: 1558-1565.

14 Viberti G, Wheeldon NM. MicroAlbuminuria Reduction With VALsartan (MARVAL) Study Investigators. Microalbuminuria reduction with valsartan in patients with type 2 diabetes mellitus: a blood pressure-independent effect. Circulation 2002; 106: 672-678.

15 Mann JF, Gerstein HC, Yi QL, Lonn EM, Hoogwerf BJ, Rashkow A, Yusuf S. Development of renal disease in people at high cardiovascular risk: Results of the HOPE randomized study. J Am Soc Nephrol 2003; 14: 641-647. 